\title{
Ka-Band LTCC Stacked Substrate Integrated Waveguide Bandpass Filter
}

\author{
Peng Zheng, ${ }^{1,2}$ Zhifu Liu $\mathbb{D}^{1},{ }^{1}$ Mingsheng Ma, ${ }^{1}$ Yi Wang $\mathbb{D},{ }^{3}$ Feng Liu, ${ }^{1}$ and Yongxiang $\mathrm{Li}^{1,4}$ \\ ${ }^{1}$ CAS Key Laboratory of Inorganic Functional Materials and Devices, Shanghai Institute of Ceramics, Chinese Academy of Sciences, \\ Shanghai 200050, China \\ ${ }^{2}$ College of Materials and Opto-Electronic Technology, The University of Chinese Academy of Sciences, Beijing 100049, China \\ ${ }^{3}$ Department of Electronic, Electrical and Systems Engineering, University of Birmingham, B15 2TT, UK \\ ${ }^{4}$ School of Engineering, RMIT University, Melbourne, VIC 3001, Australia
}

Correspondence should be addressed to Zhifu Liu; liuzf@mail.sic.ac.cn and Yi Wang; y.wang.1@bham.ac.uk

Received 24 February 2018; Accepted 29 May 2018; Published 3 July 2018

Academic Editor: Eva Antonino-Daviu

Copyright (C) 2018 Peng Zheng et al. This is an open access article distributed under the Creative Commons Attribution License, which permits unrestricted use, distribution, and reproduction in any medium, provided the original work is properly cited.

A Ka-band substrate integrated waveguide bandpass filter has been designed and fabricated using low temperature co-fired ceramic (LTCC) technology. The in-house developed SICCAS-K5F3 material with a permittivity of 6.2 and a loss tangent of 0.002 was used. The size and surface area of the proposed bandpass filter are reduced by exploiting vertical coupling in vertically laminated threedimensional structures. The coupling between adjacent cavities is realized by a narrow slot. A vertical transition structure between the coplanar-waveguide feed line and the substrate integrated waveguide is adopted to facilitate the internal signal connection. The demonstrated third-order filter has a compact size of $6.79 \mathrm{~mm} \times 4.13 \mathrm{~mm} \times 1.34 \mathrm{~mm}\left(0.63 \lambda_{0} \times 0.38 \lambda_{0} \times 0.12 \lambda_{0}\right)$ and exhibits good performance with a low insertion loss of $1.74 \mathrm{~dB}$ at $27.73 \mathrm{GHz}$ and a $3 \mathrm{~dB}$ fractional bandwidth of $10 \%$.

\section{Introduction}

Substrate integrated waveguide (SIW) has received enormous attention due to its low loss and excellent compatibility with planar circuits $[1,2]$. It preserves the advantage of rectangular waveguide and also has merits such as low profile, ease of packaging, being lightweight, and low fabrication cost. Therefore, SIW is a promising candidate structure for high performance microwave and millimeter-wave circuits. SIW is normally composed of waveguide structures defined by two rows of metallic via arrays in a dielectric substrate and sandwiched between two parallel conductor layers [3]. Apart from traditional print circuit board technology, SIW components could also be fabricated by low temperature cofired ceramic (LTCC) process. Owing to the excellent dielectric, mechanical, and thermal properties, LTCC has been widely used in high density integrated circuits package [46]. Moreover, LTCC technology is very suitable for forming stacked structures to reduce the area of the circuit.
High performance and miniaturized bandpass filter (BPF) is an essential component in modern wireless communication system. With a BPF, the required channel spectrum is selected while the unwanted spectrum is rejected. Various BPFs are implemented by diverse transmission line technologies such as microstrip [7] and SIW [8]. Direct-coupled or cross-coupled SIW cavities are common in millimeter-wave filter designs $[9,10]$. Compared with in-plane arranged SIW cavities, multilayer stacked SIW cavities are more compact.

In the design of high frequency transmission circuits, the internal signal connection and transition are vitally important, especially in multilayer LTCC package. Generally, the transition takes either horizontal or vertical form. Coplanarwaveguide- (CPW)- to-stripline vertical transitions have been researched extensively in recent years. In previous report, a CPW-to-stripline vertical transition was utilized for a V-band LTCC system-on-package applications [11]. A broadband CPW-to-stripline transition which is capable of operation up to $100 \mathrm{GHz}$ was also presented for LTCC 


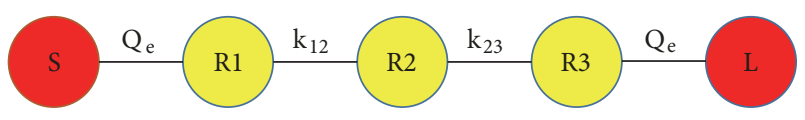

FIGURE 1: Coupling topology of the proposed filter.

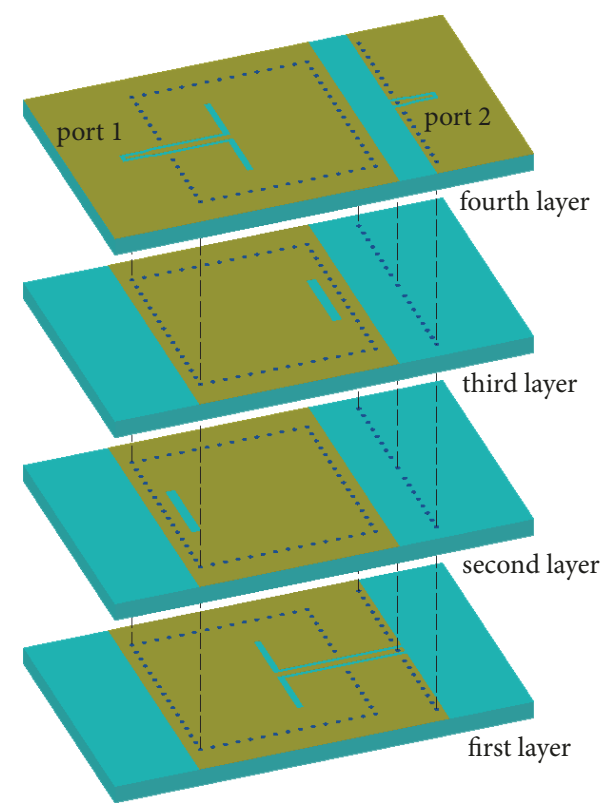

FIGURE 2: Exploded view of the proposed LTCC BPF.

module [12]. However, few works were reported on the CPWto-CPW vertical transition for LTCC based SIW BPF.

In this paper, a compact Ka-band LTCC SIW BPF with a via-based CPW-to-CPW vertical transition is presented. The SIW cavities are vertically stacked to allow miniaturization. As a key element of the circuit integration, the CPW transition is adopted and analyzed. To validate the proposed scheme, a third-order SIW BPF in Ka-band was designed and fabricated using LTCC technology. The simulation result and measurement result are in good agreement. Competitive performance has been shown as compared with other multilayer SIW filters. It should also be noted that an in-house developed LTCC material was used in this work.

\section{BPF Design}

2.1. Overview of Structure. The coupling topology of the proposed third-order SIW BPF is depicted in Figure 1. S represents the source excitation, $L$ represents the Load, and $R 1, R 2$, and $R 3$ represent three SIW resonant cavities. $Q_{e}$ is the external quality factor. $k_{12}$ and $k_{23}$ are the coupling coefficients.

The architecture of the proposed LTCC BPF is illustrated in Figure 2. The third-order BPF is built in four layers of LTCC substrates and four layers of metallization. The three resonators are vertically coupled to each other through slots which are made in the metallization layers and close to the wall. The coupling between adjacent SIW resonant cavities is magnetic in nature. A CPW structure is adopted as the excitation ports for measurements using the common ground-signal-ground (GSG) probe. In order to bring the two CPW ports to the same circuit level, a CPW-to-CPW vertical transition structure at port 2 is used to facilitate the measurement.

The dielectric substrates are based on the SICCAS-K5F3 LTCC material with a permittivity of 6.2 and a loss tangent of 0.002. This material has been in-house developed at Shanghai Institute of Ceramics, Chinese Academy of Sciences. The thickness of each layer in this design is $0.3 \mathrm{~mm}$, formed by 10 sheets of green tapes. $10 \mu \mathrm{m}$ silver layer is screen-printed on the LTCC substrates acting as the top and bottom enclosure planes for the cavities.

2.2. SIW Resonant Cavity. The resonant cavity is composed of laminated dielectric substrate, parallel conductor layers, and metal via arrays. To design a SIW cavity operating at a given frequency, the following formula is adopted.

$$
\begin{aligned}
f_{\text {res }} & =\frac{c}{2 \pi \sqrt{\varepsilon_{r}}} \sqrt{\left(\frac{m \pi}{L_{e f f}}\right)^{2}+\left(\frac{n \pi}{W_{e f f}}\right)^{2}} \\
L_{e f f} & =L-\frac{d^{2}}{0.95 p} \\
W_{e f f} & =W-\frac{d^{2}}{0.95 p}
\end{aligned}
$$

where $c$ is the speed of light in vacuum and $\varepsilon_{r}$ is the dielectric constant of the LTCC substrate. $L$ and $W$ are the length and width of the SIW resonant cavity and $d$ and $p$ represent the diameter of the metal via and the periodic length of the metal via array, respectively. Based on these formulas, the initial dimensions of the SIW cavity are determined.

2.3. Coupling Slots and Feeding Structure. According to the general Chebyshev BPF theory, the normalized coupling coefficients and the external quality factors can be obtained by following formula.

$$
\begin{aligned}
\mathrm{Q}_{\mathrm{e} 1} & =\frac{\mathrm{g}_{0} \mathrm{~g}_{1}}{\mathrm{FBW}}, \\
\mathrm{Q}_{\mathrm{en}} & =\frac{\mathrm{g}_{0} \mathrm{~g}_{1}}{\mathrm{FBW}}, \\
\mathrm{M}_{\mathrm{i}, \mathrm{i}+1} & =\frac{1}{\sqrt{\mathrm{g}_{\mathrm{i}} \mathrm{g}_{\mathrm{i}+1}}}, \text { for } \mathrm{i}=1 \text { to } \mathrm{n}-1
\end{aligned}
$$

The presented third-order Chebyshev BPF is designed with following specifications: passband return loss, $20 \mathrm{~dB}$; fractional bandwidth (FBW), 6\%. The low-pass prototype values can be determined as $\mathrm{g}_{0}=\mathrm{g}_{4}=1, \mathrm{~g}_{1}=\mathrm{g}_{3}=0.8535$, and $g_{2}=1.1039$. The external quality factors are calculated as follows: $\mathrm{Q}_{\mathrm{e} 1}=\mathrm{Q}_{\mathrm{en}}=14.225$. The coupling coefficients are $\mathrm{k}_{12}=\mathrm{k}_{23}=\mathrm{FBW} \times \mathrm{M}_{12}=0.0618$.

To minimize the occupation area, the resonant cavities are vertically stacked rather than cascaded laterally. The coupling between adjacent SIW cavities is realized by a narrow slot 


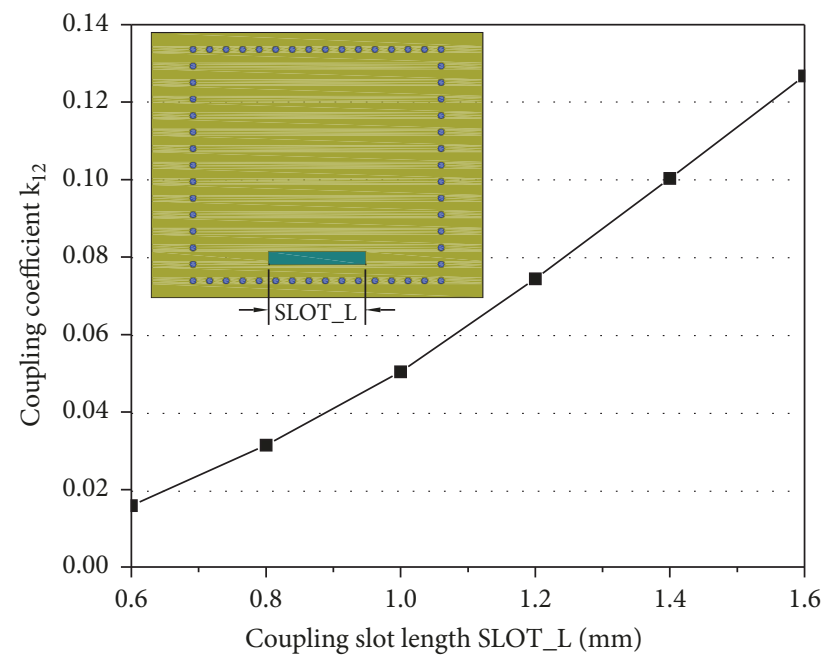

FIGURE 3: Relationship between the coupling coefficients $\mathrm{k}_{12}$ and the coupling slot length SLOT_L.

close to the metal via array. Since the SIW cavities are coupled together via the magnetic slot, the central frequency peak of the SIW cavities splits into two discrete peaks with frequencies $f_{1}$ and $f_{2}$, respectively. The lower resonant frequency point $f_{1}$ and the higher frequency point $f_{2}$ are obtained from the simulation by ANSYS HFSS. The coupling coefficient $\mathrm{k}_{12}$ between the adjacent cavities is extracted using the following formula:

$$
\mathrm{k}_{12}=\frac{f_{2}^{2}-f_{1}^{2}}{f_{2}^{2}+f_{1}^{2}}
$$

With the combination of the formula and ANSYS HFSS simulation, the coupling coefficient related to the slot dimension is achieved. The relationship between the extracted coupling coefficient and coupling slot length SLOT_L is depicted in Figure 3. When the coupling slot length is increased, the coupling coefficients become larger. Accordingly, the coupling coefficients can be tuned by the length, width, and position of the slot. The final values have been achieved by optimization using the full-wave simulator.

The external quality factor $Q_{e}$ depends on the input and output excitation structure. This structure is composed of a section of $50 \mathrm{ohm} \mathrm{CPW} \mathrm{line} \mathrm{and} \mathrm{a} \mathrm{slot} \mathrm{dipole} \mathrm{for} \mathrm{coupling} \mathrm{to}$ the SIW cavity as shown in the inset of Figure 4. The external quality factor $Q_{e}$ can be extracted using following formula:

$$
Q_{e}=\frac{f_{0}}{\Delta f_{ \pm 90^{\circ}}}
$$

where $f_{0}$ is the resonant frequency of the structure shown in Figure 4 and $\Delta f_{ \pm 90^{\circ}}$ is the frequency bandwidth corresponding to the phase shift of $\pm 90^{\circ}$ with respect to the absolute phase at $f_{0}$. Using ANSYS HFSS simulation, the external quality factor $Q_{e}$ can be derived. The external quality factor of the feeding structure is determined by the slot dipole length, width, and offset position. The relationship between the external quality factor and the slot dipole offset S_OFFSET is

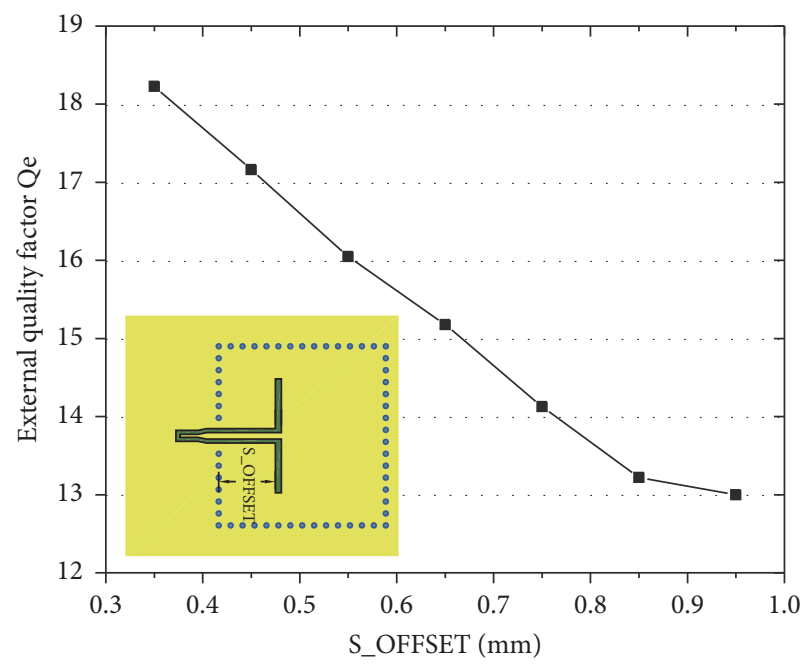

FIGURE 4: Relationship between the external quality factor $Q_{e}$ and the offset value S_OFFSET.

depicted in Figure 4. When the S_OFFSET value is increased, the external quality factor becomes smaller. The final values have been achieved by optimization in ANSYS HFSS.

2.4. CPW-to-CPW Vertical Transitions. Microwave and millimeter-wave component measurement are often accomplished using GSG on-wafer probes. The back-to-back CPW structure is commonly utilized. As illustrated in Figure 5, the proposed CPW-to-CPW vertical transition structure is composed of two sets of metal vias and three sections of CPW transmission lines using a two-layer LTCC substrate. The thickness of the top layer is $0.1 \mathrm{~mm}$; the thickness of the bottom layer is $0.9 \mathrm{~mm}$. The diameter of the via hole is $0.09 \mathrm{~mm}$ and the metal used is silver.

To maintain a good transmission and reflection characteristics, the CPW line is designed to be $50 \mathrm{ohm}$ in Ka band. The width of the signal line is $0.25 \mathrm{~mm}$, and the gap is 0.066 $\mathrm{mm}$. The structure is simulated with ANSYS HFSS software. As depicted in Figure 6, the CPW vertical transition structure has a return loss better than $15 \mathrm{~dB}$ from 20 to $36 \mathrm{GHz}$. The simulated insertion loss is less than $0.61 \mathrm{~dB}$.

Based on the above analysis, the CPW-to-CPW vertical transition structure is adopted at port 2 and optimized to miniaturize the multilayer SIW filter. Figure 7 shows the simulated performance of the whole SIW filter. It is observed that the length of the CPW line has a noticeable impact on the reflection coefficients. This is mainly caused by the imperfect impedance matching due to the introduction of the vertical transition structure. When the CPW line length is 0.6 $\mathrm{mm}$, the return loss is over $20 \mathrm{~dB}$. After full-wave simulation and optimization, the final dimensional parameters of the proposed SIW BPF are presented in Figure 8 and Table 1.

\section{Fabrication and Measurement}

3.1. LTCC Fabrication Process. The dielectric material used during the LTCC fabrication process was SICCAS-K5F3 developed by Shanghai Institute of Ceramics, Chinese 


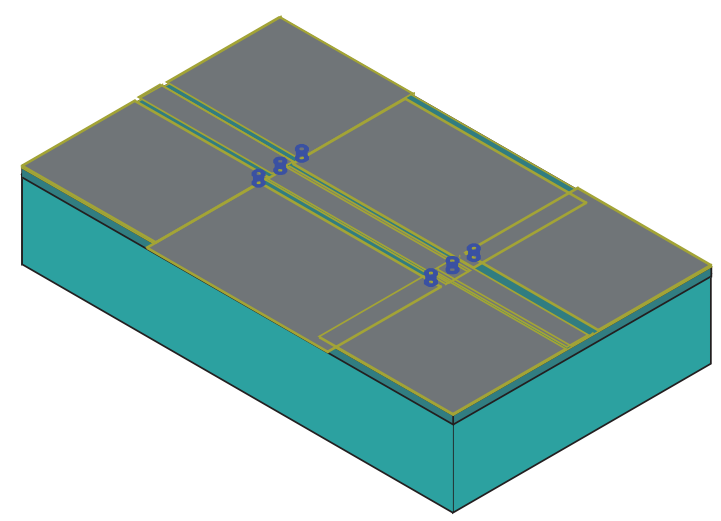

FIGURE 5: The model of the proposed CPW vertical transition structure.

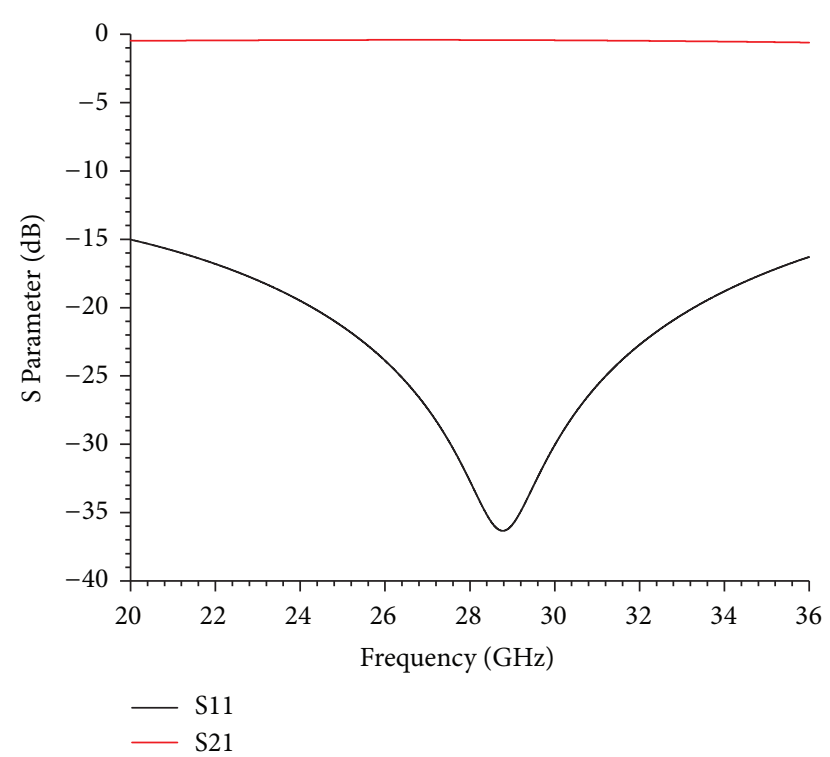

FIGURE 6: Simulated S-parameters of the CPW vertical transition.

TABLE 1: Dimensions of the SIW filter (mm).

\begin{tabular}{lcccc}
\hline S_L & S_W & SIW_L & SIW_W & VIA_R \\
\hline 6.7 & 4.0 & 2.8 & 3.0 & 0.04 \\
\hline VIA_P & H & SLOT_L & SLOT_W & SLOT_OFFSET \\
\hline 0.2 & 1.2 & 1.17 & 0.15 & 0.3 \\
\hline CP_L & CP_W & CPW_W & CPW_C & CPW_G \\
\hline 0.9 & 0.1 & 0.1 & 0.6 & 0.067 \\
\hline
\end{tabular}

Academy of Sciences. The SICCAS-K5F3 green tape has a thickness of $50 \mu \mathrm{m}$. Holes with $0.1 \mathrm{~mm}$ diameter were punched by a KEKO, PAM-200 equipment. Silver paste (Dupont LL612) was deposited on the LTCC green tape by screen printing technique (KEKO, P-200 equipment). The vias were filled with silver paste. The green tapes were staked in proper order and laminated at $7000 \mathrm{psi}$ and $70^{\circ} \mathrm{C}$ for $25 \mathrm{~min}$ in an isostatic pressing chamber (LT08001, PTC). Small filter blocks were obtained by cutting the pressed green block. The
TABLE 2: SIW bandpass filters comparison.

\begin{tabular}{lccccc}
\hline Ref & Order & $f_{0}(\mathrm{GHz})$ & Insertion Loss $(\mathrm{dB})$ & FBW & Area $\left(\lambda_{0}{ }^{2}\right)$ \\
\hline$[4]$ & 4 & 27.95 & 2.8 & $9 \%$ & $0.57 \times 0.57$ \\
{$[13]$} & 2 & 34.5 & 1.7 & $2.5 \%$ & $0.5 \times 0.5$ \\
{$[14]$} & 4 & 35 & 1.25 & $3.6 \%$ & $1.28 \times 1.28$ \\
{$[15]$} & 4 & 29.87 & 3.3 & $13 \%$ & $0.37 \times 0.28$ \\
{$[16]$} & 2 & 27.6 & 1.8 & $2.5 \%$ & $0.78 \times 0.77$ \\
This work & 3 & 27.7 & 1.74 & $10 \%$ & $0.63 \times 0.38$ \\
\hline
\end{tabular}

filters were obtained after debinding at $450^{\circ} \mathrm{C}$ for $2 \mathrm{~h}$ and sintered at $900^{\circ} \mathrm{C}$ for $25 \mathrm{~min}$. Owing to the shrinkage in the process, the designed value and fabricated dimension had a slight discrepancy. The photograph of the fabricated filter is exhibited in Figure 9(a), while the microscopic image of the CPW port is presented in Figure $9(\mathrm{~b})$. The fabricated filter is 6.79 $\mathrm{mm} \times 4.13 \mathrm{~mm} \times 1.34 \mathrm{~mm}\left(0.63 \lambda_{0} \times 0.38 \lambda_{0} \times 0.12 \lambda_{0}\right)$ in size.

3.2. Measurement. The LTCC SIW filter sample was measured using a Keysight N5245A Vector Network Analyzer (VNA) and a Cascade M150 probe station with two GSG probes after a SOLT calibration. The measured and simulated S-parameters of the SIW filter are plotted in Figure 10. The measurement results are in good agreement with the simulation results. The measured insertion loss is $1.74 \mathrm{~dB}$ at the center frequency of $27.73 \mathrm{GHz}$. The $3 \mathrm{~dB}$ bandwidth of the filter is $10 \%$ from $26.30 \mathrm{GHz}$ to $29.08 \mathrm{GHz}$. Some discrepancies were observed in the return loss and transmission at the upper band edge. These are mainly due to the fabrication tolerance. The performance of the SIW BPF in this work, as compared with a few other reported results, is presented in Table 2. It is demonstrated that the SIW filter has a compact size, low insertion loss, and good comparable bandwidth.

\section{Conclusions}

In this paper, a Ka-band multilayer SIW BPF with CPW-toCPW vertical transition is designed and fabricated using an in-house developed LTCC material and process. The coupling is realized by inductive slots between different layers. The CPW-to-CPW vertical transition structure is analyzed and 


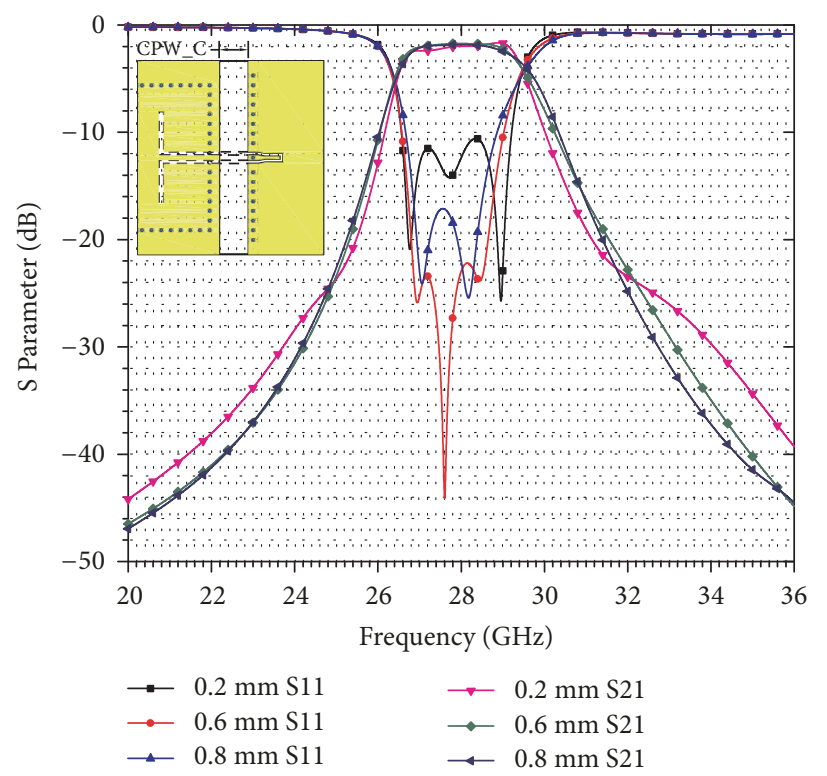

FIGURE 7: Effect of the length of the CPW line on the S-parameters of the SIW filter.

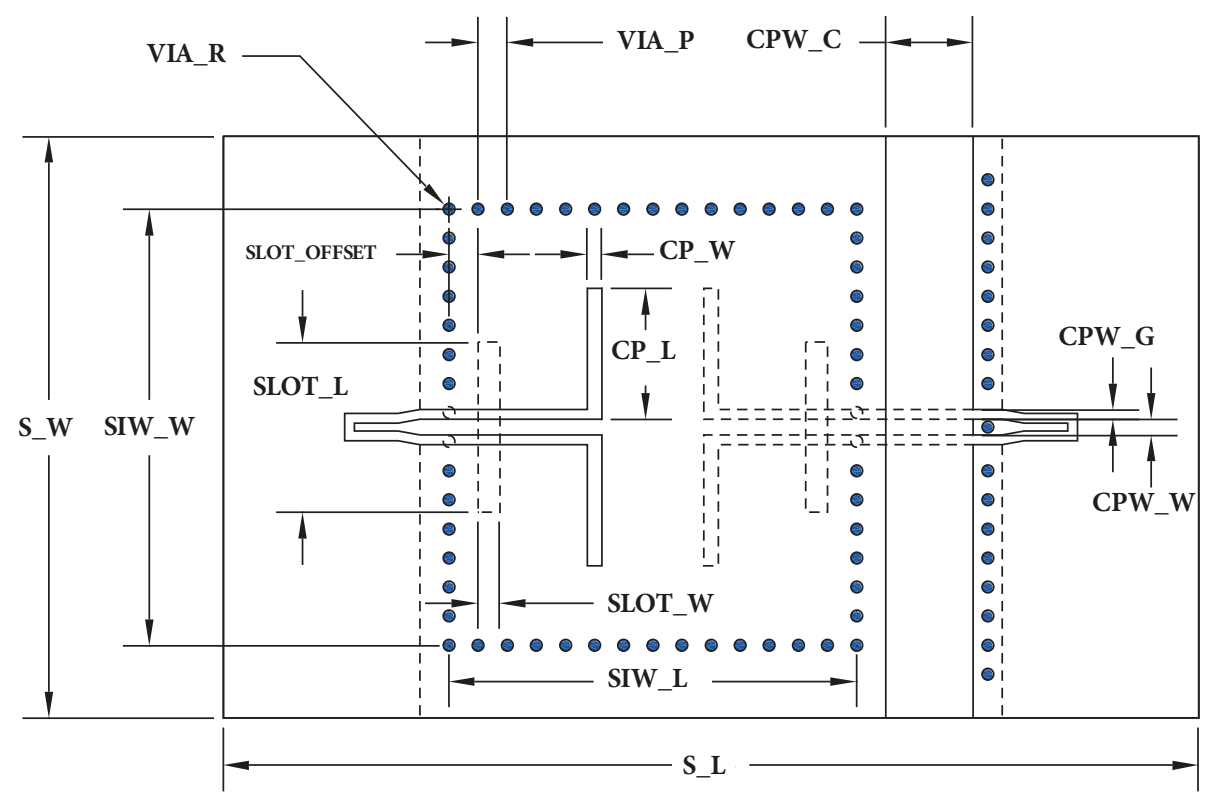

FIGURE 8: Geometric parameters of the proposed filter.

implemented in the multilayer filter. The simulated and measured BPF results exhibit good performance and agree reasonably well. The fabricated SIW filter has a center frequency of $27.73 \mathrm{GHz}$, low insertion loss of $1.74 \mathrm{~dB}$, and a $3 \mathrm{~dB}$ FBW of about $10 \%$. The competitive performance of the SIW filter also validates the LTCC materials used in this work.

\section{Data Availability}

The data used to support the findings of this study are available from the corresponding author upon request.

\section{Conflicts of Interest}

The authors declare that they have no conflicts of interest.

\section{Acknowledgments}

The authors would like to acknowledge the financial support from the National Key Research and Development Program of China (2017YFB0406303), the National Natural Science Foundation of China (61501438), and the Youth Innovation Promotion Association of CAS. 


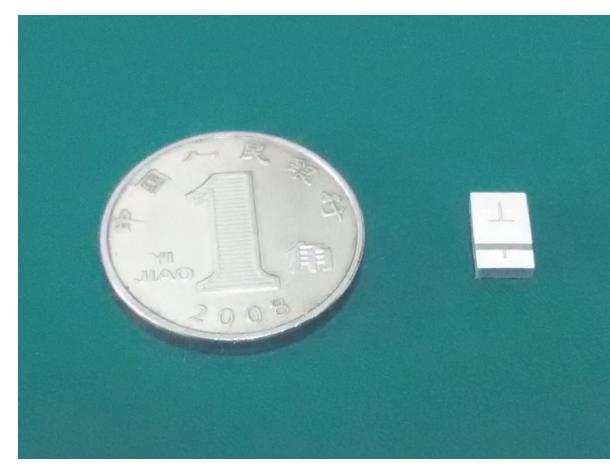

(a)

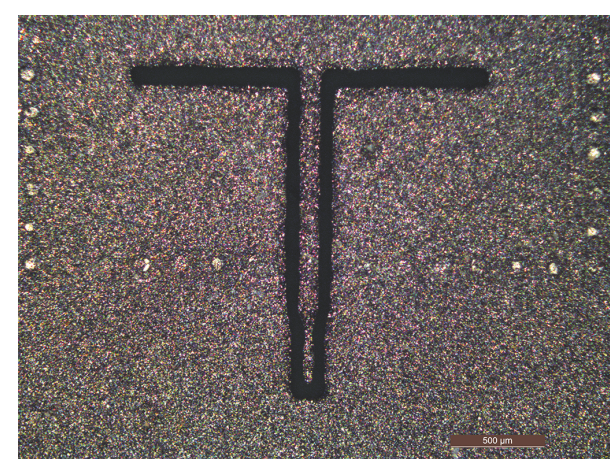

(b)

Figure 9: Photograph of (a) the filter sample and (b) the CPW transmission line at the input port.

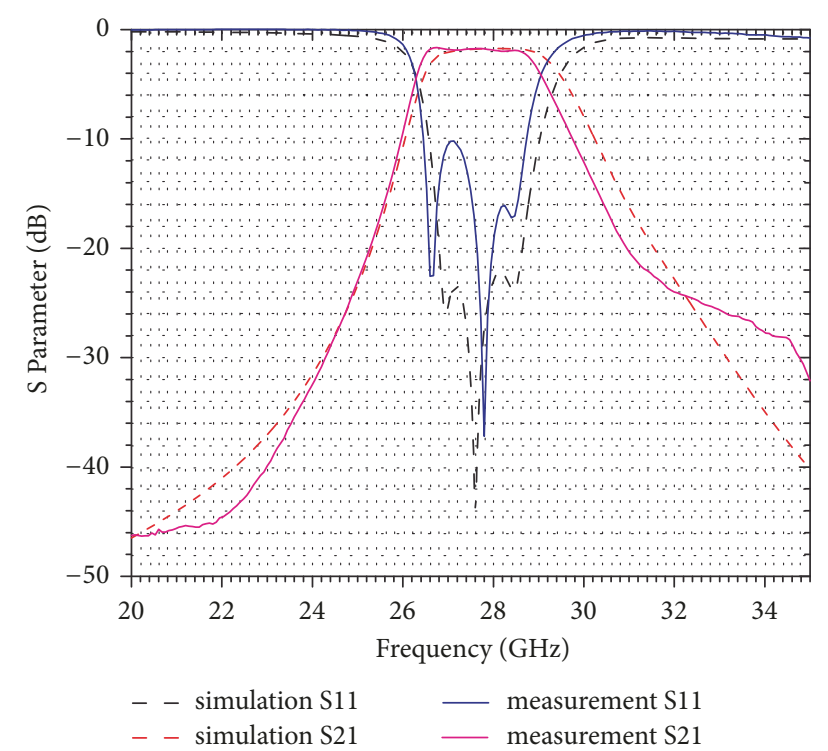

FIgURE 10: Simulation and measurement results of the proposed filter.

\section{References}

[1] X.-P. Chen and K. Wu, "Substrate integrated waveguide filters: design techniques and structure innovations," IEEE Microwave Magazine, vol. 15, no. 6, pp. 121-133, 2014.

[2] C. Yu and W. Hong, "37-38 GHz substrate integrated filtenna for wireless communication application," Microwave and Optical Technology Letters, vol. 54, no. 2, pp. 346-351, 2011.

[3] D. Deslandes and K. Wu, "Accurate Modeling, Wave Mechanisms, and Design Considerations of a Substrate Integrated Waveguide," IEEE Transactions on Microwave Theory and Techniques, vol. 54, no. 6, pp. 2516-2526, 2006.

[4] K. S. Chin, C. C. Chang, C. H. Chen, Z. Guo, D. Wang, and W. Che, "LTCC Multilayered Substrate-Integrated Waveguide Filter With Enhanced Frequency Selectivity for System-in-Package Applications," IEEE Transactions on Components Packaging ¿amp; Manufacturing Technology, vol. 4, no. 4, pp. 664-672, 2014.

[5] S. W. Wong, K. Wang, Z.-N. Chen, and Q.-X. Chu, "Electric coupling structure of substrate integrated waveguide (SIW) for the application of 140-GHz bandpass filter on LTCC," IEEE Transactions on Components, Packaging, and Manufacturing Technology, vol. 4, no. 2, pp. 316-322, 2014.

[6] I. Llamas-Garro, F. Mira, P. Zheng, Z. Liu, L.-S. Wu, and Y. Wang, "All-resonator based LTCC diplexer using substrateintegrated-waveguides," IEEE Electronics Letters, vol. 53, no. 21, pp. 1410-1412, 2017.

[7] S. Zhang and L. Zhu, "Compact and high-selectivity microstrip bandpass filters using triple-/quad-Mode stub-loaded resonators," IEEE Microwave and Wireless Components Letters, vol. 21, no. 10, pp. 522-524, 2011.

[8] X.-P. Chen and K. Wu, "Substrate integrated waveguide crosscoupled filter with negative coupling structure," IEEE Transactions on Microwave Theory and Techniques, vol. 56, no. 1, pp. 142-149, 2008.

[9] Q.-F. Wei, Z.-F. Li, L. Li, W.-J. Zhang, and J.-F. Mao, “Threepole cross-coupled substrate-integrated waveguide bandpass filters based on PCB process and multilayer LTCC technology," Microwave and Optical Technology Letters, vol. 51, no. 1, pp. 7173, 2009.

[10] S. Sirci, M. Á. Sánchez-Soriano, J. D. MartíNez et al., "Design and Multiphysics Analysis of Direct and Cross-Coupled SIW Combline Filters Using Electric and Magnetic Couplings," IEEE Transactions on Microwave Theory \& Techniques, vol. 63, no. 12, pp. 4341-4354, 2015.

[11] Y. C. Lee, "CPW-to-stripline vertical via transitions for $60 \mathrm{GHz}$ LTCC SOP applications," Progress in Electromagnetics Research Letters, vol. 2, pp. 37-44, 2008.

[12] D. M. Nair, W. E. McKinzie III, B. A. Thrasher, M. A. Smith, E. D. Hughes, and J. M. Parisi, "A $10 \mathrm{MHz}$ to $100 \mathrm{GHz}$ LTCC CPWto-stripline vertical transition," in Proceedings of the 2013 IEEE MTT-S International Microwave Symposium Digest, MTT 2013, June 2013.

[13] M. Shen, Z. Shao, X. Du, Z. He, and X. Li, "Ka-band multilayered substrate integrated waveguide narrowband filter for system-in-package applications," Microwave and Optical Technology Letters, vol. 58, no. 6, pp. 1395-1398, 2016.

[14] X.-P. Chen and K. Wu, "Self-packaged millimeter-wave substrate integrated waveguide filter with asymmetric frequency response," IEEE Transactions on Components, Packaging, and Manufacturing Technology, vol. 2, no. 5, pp. 775-782, 2012.

[15] T.-M. Shen, T.-Y. Lin, T.-Y. Huang, and R.-B. Wu, "A vertically stacked quasi-elliptic waveguide filter with crossly coupling 
vias," in Proceedings of the Asia-Pacific Microwave Conference, APMC 2007, December 2007.

[16] D. Deslandes and K. Wu, "Millimeter-Wave Substrate Integrated Waveguide Filters," in Proceedings of the CCECE 2003 Canadian Conference on Electrical and Computer Engineering: Toward a Caring and Humane Technology, pp. 1917-1920, May 2003. 


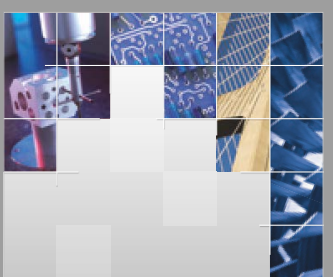

\section{Enfincering}
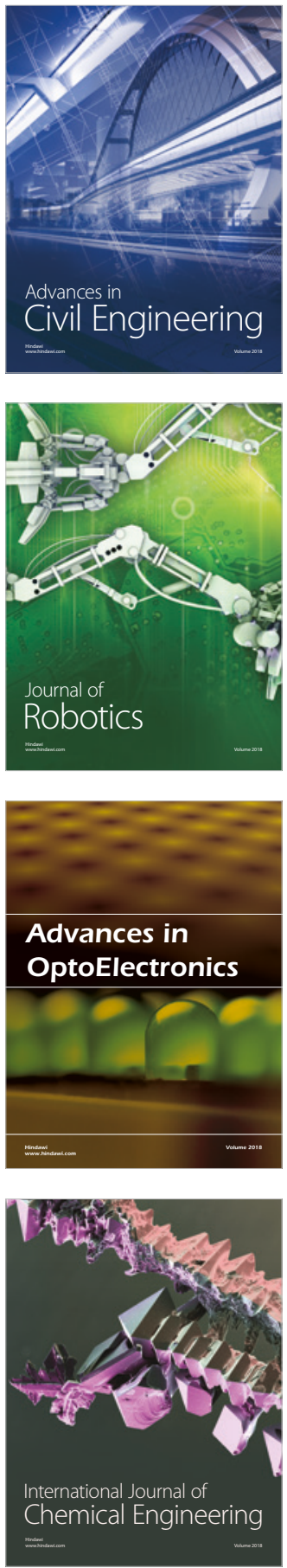

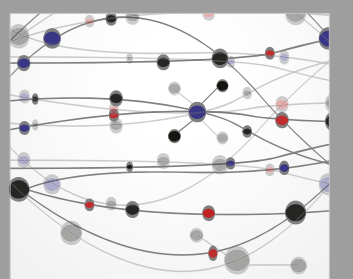

\section{Rotating \\ Machinery}

The Scientific World Journal

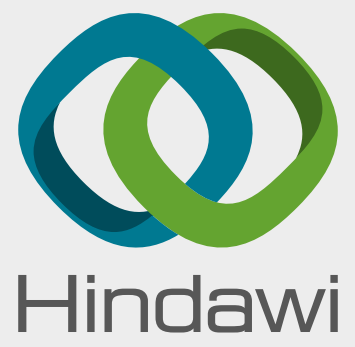

Submit your manuscripts at

www.hindawi.com
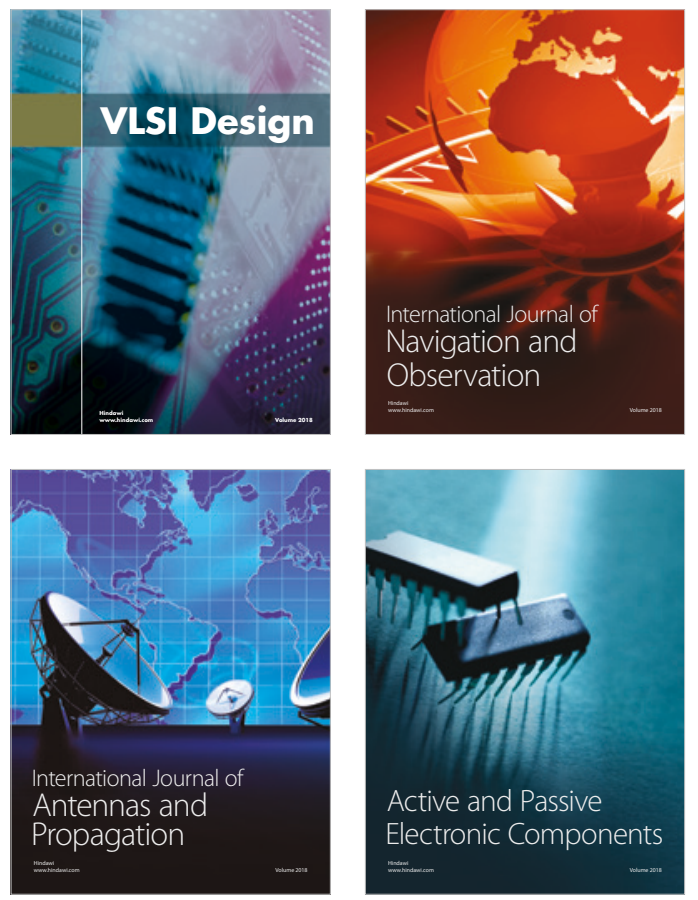
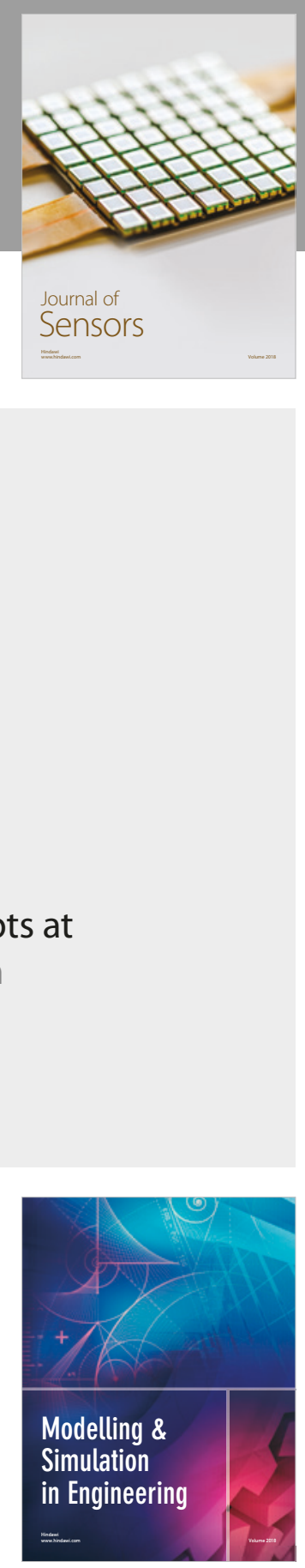

\section{Advances \\ Multimedia}
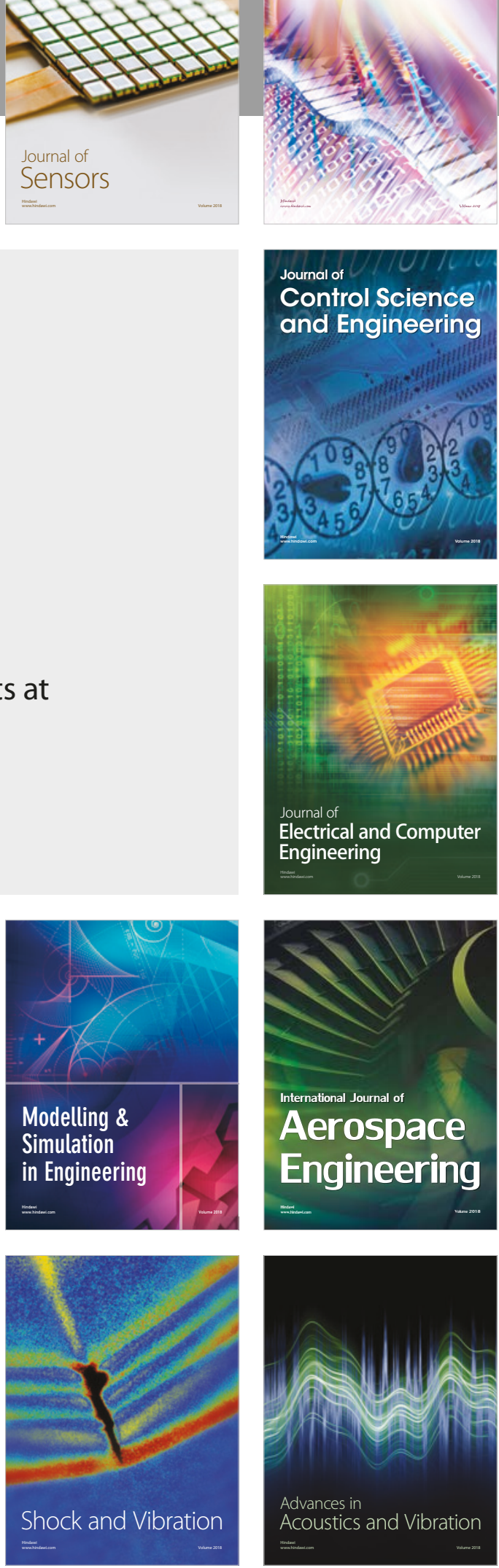